Voix et Images

voixetimages

\title{
Exploitation et utilisation des paramètres : Ducharme, Thériault
}

\section{Jean-Claude Choul}

Volume 7, numéro 3, printemps 1982

Anne Hébert

URI : https://id.erudit.org/iderudit/200349ar

DOI : https://doi.org/10.7202/200349ar

Aller au sommaire du numéro

Éditeur(s)

Les Presses de l'Université du Québec

ISSN

0318-9201 (imprimé)

1705-933X (numérique)

Découvrir la revue

Citer cet article

Choul, J.-C. (1982). Exploitation et utilisation des paramètres : Ducharme, Thériault. Voix et Images, 7(3), 571-579. https://doi.org/10.7202/200349ar d'utilisation que vous pouvez consulter en ligne.

https://apropos.erudit.org/fr/usagers/politique-dutilisation/ 


\title{
Exploitation et utilisation des paramètres: Ducharme. Thériault
}

\author{
par Jean-Claude Choul, Université Dalhousie. Halifax
}

On sait le rôle de l'incipit dans la mise en place de l'extra-texte, qui tout comme le titre participe à un certain réglage du discours et à la détermination de l'axe thématique d'une narration'.

Dans une recherche antérieure, j'ai moi-même parcouru la série d'hypothèses proposant de voir un programme dans le texte, dont la fonction première serait de régler la lecture et de l'asservir par des concaténations de signaux ${ }^{2}$. Parmi ces signaux dont le décodage est obligatoıre pour satısfaire aux exigences minimales du texte, j'ai pu en indiquer un certain nombre qui gagnent à être identifiés comme des paramètres, c'est-à-dire des constantes de discours et qui varieraient avec le type de discours. à l'égal des schèmes ou programmes narratifs ${ }^{3}$.

- Par décodage obligatorre, il faut entendre que le lecteur doit disposer d'une compétence idéologico-littéraıre qui lui permettra de mener à bien son entreprise lectrice, entre la consommation pure et simple et la production. II est donc censé posséder une "bibliotheqque imaginaire" sous forme de code labile et dont les éléments lui faciliteront la reconnaissance du matériau auquel il est confronté.

La lecture oscille aınsi entre deux pôles: l'utilisation caractérisée par la très grande manipulation (participation) exigée du lecteur et la communication où la piupart des opérations sont automatisées. Ces deux pôles permettent de poser une échelle de lisibilité, le lisible étant du côté de la communication. et le scriptible du côté de l'utilisation.

II est entendu que cette échelle varie également en fonction des compétences du lecteur. II n'y a pas de lisıbilité absolue d'un texte. Un premier exemple nous est donné par la phrase d'ouverture de La Mort d'eau d'Y. Thériault ${ }^{4}$ :

Il filait les $i$.

Si cette réalisation phrastique est reconnue, elle ne le sera que par un lecteur disposant dans son univers de discours d'une unité de code (codème) du type /filer (une note) $=$ chanter en faisant varier l'intensité. 
On comparera la phrase d'ouverture de L'Océantume de R. Ducharme, qui pour être décodée de façon satisfaisante n'exige aucune compétence spécialisée ${ }^{5}$ :

Elle me demande si j'ai bien appris mes leçons, puis elle me crie d'aller me coucher: comme à un chien.

Cette remarque appelle une précision théorique : le modèle de réception auquel nous nous rapportons lorsqu'il est question du lecteur fait état de trois univers distincts, mais liés entre eux: l'univers de discours, de type lexicosémantique, l'univers de coordonnées, de type pragmatico-narratif, et l'univers de signification, de type axiologique où les modeles narratifs et les données encyclopédiques sont évalués.

Des trois univers, le plus important pour la narratologie est certainement le second où se constituent les référentiels successifs des textes, notamment par anaphorisation, comme l'illustre la deuxième phrase de L'Océantume (OCE par la suite):

La maîtresse d'école a des dents en or plein la bouche.

Il est clair que dans un traitement automatisé le lecteur va remplir la place vide du elle de la phrase précédente par la maîtresse d'école, mais cet exemple permet également d'indiquer que si cet énoncé ne pose pas de difficultés de décodage lexico-sémantique, il n'en va pas de même quant à l'articulation des éléments du référentiel qui se construit au cours de la lecture.

On peut donc imaginer qu'il existe un type d'illisibilité lié à l'univers de coordination, et qui ne réside pas seulement dans le choix des scénarios disponibles, mais aussi dans leur enchainement. L'anaphorisation (cataphorique) de elle sera par exemple modifiée par une manipulation supplémentaire dans la suite de la lecture.

Toutefois, ce n'est pas l'anaphorisation qui nous retiendra ici, mais il faut noter quelle constitue un des mécanismes fondamentaux de la lecture, concaténant entre elles les constantes discursives, par les places que celles-ci comportent.

Ainsi, // filait les $i$, attend un /sujet humain/. Babin, qui lui assure la suite du texte. Les constantes discursives qui suivent présentent dans La Mort d'eau (MOE par la suite) une homogénéité d'anaphorisation:

(II) mettre un accent circonflexe

(II) dire ainsi

(II) être + origine (Madelinot)

Cette uniformité facilite la constitution du rôle Agent du référentiel, qui demeure un problème dans l'OCE, de par l'intercalation d'un candidat qui devra ètre rejeté.

La régularité d'anaphorisation de l'incipit de la Passe-au-crachin d'Y. Thériault (PAC par la suite), malgré l'introduction successive de trois person. 
nages est caractéristique de la stabilité probable du référentiele.

Contrairement à ce qu'un premier contact pourrait laisser supposer, il n'y a pas a priori de corrélation entre le nombre de paramètres d'un texte et sa stabilité référentielle. Il est du moins impossible de poser un règle de proportionnalité.

On voit ainsi lintérêt qu'il y a à parler de constantes discursives et de paramètres plutôt que de clichés.

La notion de cliché (figement phraséologique) se prête difficilement à l'analyse et à la description des textes en raison de sa charge connotative. Il est trop simple, par exemple, d'assimiler cliché et lisibilité ou encore cliché et stabilité référentielle (adéquation cliché et stéréotype).

Si /filer une note/ constitue un codème, le décodage de /l filait les i, ne peut faire l'économie d'une manipulation, même si la suite du texte fonctionne comme explicitation:

II leur mettait un accent circonflexe. İl disait "les firiilles"

De même l'incipit de PAC, dans son ordre naturel apparent, constitutif d'un déroulement quasi stéréotypique, est fondé sur une manipulation propre à l'univers de signification, où le détail algorithmique du programme /dépecer le poisson/ va être subsumé par un paramètre fourni par le texte : c'était une tâche d'homme, sert à démarquer le personnage de Marie.

- Cette remarque permet d'assigner au paramètre l'une de ses fonctions dans la lecture (et le texte): il rend possible économiquement la synthèse de la narration, en coiffant les expansions algorithmiques du discours et simplifie les manipulations de l'univers de signification.

Le paramètre a également une fonction régulatrice dans l'établissement d'une cohérence lexicale, ainsi dans PAC:

en toute honnèteté

en tant que marque de rejet, corrige les inférences qu'entraine l'apparition d'une autre constante (de type locutionnel): lever la main.

On observe un phénomène analogue dans OCE où un peu comme fonctionne comme marque de relativisation de la comparaison:

IIs l'ont cimenté (le bateau) dans le sol, un peu comme une pierre tombale.

Le caractère quasi imprévisible de la comparaison et sa relative incohérence sont ainsi normalisés.

II faut toutefois signaler que dans OCE la cohérence lexico-sémantique n'est pas le corollaire obligé de l'emploi des paramètres. En effet si le je souhaite qu'ilchavire présente une cohérence sémantique par rapport au bateau cimenté (chavirer fait partie des propriétés d'un bateau), il n'en va pas tout a fait de même pour cet extrait: 
Nous sommes partis en voyage et, quand nous sommes revenus, les rats avaient mangé tout le château, sauf la pierre.

ou

Parfois la nuit, toute la terre de l'île se change en rats et les restes du château chancellent.

ou encore

Je m'entortille comme un bonbon dans mes couvertures.

L'interprétation de ces énoncés, que l'on pourrait considérer comme non immediate, se fera pourtant au moyen de paramètres. Si / chavirer/peut figurer dans la.contiguïté de /bateau/, il en va de même pour /cartes/ dans la contiguité de /château/, mème si la probabilité semble moins grande.

De même, la mutation de la terre en rats peut se voir assigner une interprétation partielle ou provisoire à partir d'énoncés mémorisés du type /la nuit tout est possible/ ou encore /délire onirique/que confirmerait l'énoncé normalisateur: lode; va te coucher.

La comparaison emballage-bonbon/lode-couverture est rétablie et avalisée elle aussi au moyen d'un paramètre: entortiller un bonbon.

On notera que OCE, dans un rapprochement avec PAC ou MOE, présente une relative complexité de manipulations et on peut supposer que ces difficultés apparentes (aspérités du texte) peuvent retarder la constitution d'un référentiel, dont l'économie intrinsèque constitue sa validité pour l'ensemble du texte ${ }^{7}$.

En effet, une des conditions de la lisibilité d'un texte se trouve non seulement dans la stabilité de ses référents, mais aussi dans la permanence ou la pérennité de ceux-ci. Sans nécessairement faire allusion à la règle des trois unités, si les coordonnées spatio-temporelles demeurent les mèmes pour toute l'étendue d'un texte, celui-ci gagnera en lisibilité, puisque le lecteur n'aura pas à en élaborer de nouvelles (économie de manipulations).

II est clair, en outre, que l'exploitation des paramètres par le texte caractéristique de OCE correspond à la nécessité où se trouve le lecteur de procéder à un plus grand nombre de manipulations.

Cependant, on évitera de généraliser, car MOE présente aussi quelques problèmes de lisibilité dès l'incipit, qui eux aussi peuvent retarder la mise en place du référentiel ou en rendre l'élaboration difficile.

En effet, un certain nombre des paramètres qu'on peut relever appartient au lexique de la musique, ou exploite celui-ci : mème /marquer/dans:

Il disait aussi «câlice» et cela le marquait

peut être rapporté à ce lexique, avec:

une valeur de soupir

une phrase d'actes quotidiens 
En fait, le passage' est caractéristique de ce que nous désignons par exploitation des paramètres. Ainsi, nous sommes en présence d'une double sollicitation, code musical et code typographique :

C'était un soupir, une valeur de soupir, ou de ponctuation. Un point et virgule dans une phrase d'actes quotidiens.

On remarque que c'est /ponctuer/ qui réalise la fusion des deux zones distinctes de l'univers de discours.

Ces marques du texte vont être synthétisées par le texte lui-mème, au moyen d'un autre paramètre, qui va coiffer le passage:

ils chantaient eux aussi en parlant.

Ce phénomène déjà observé dans PAC avec c'était une tâche d'homme, adopte une modalité quelque peu différente dans OCE, mais se modèle tout de même sur un paramètre s'imaginer que:

Je m'imagine que l'école brûle. Je m'imagine que j'ai des dents en or comme la maitresse et je suce mes dents.

Outre la fonction normalisatrice du paramètre en raison de son caractère explicatif (dans les trois textes examinés), il est plausible d'y voir comme une ponctuation de l'activité de lecture, une sorte de pause correspondant a l'élaboration, chez le lecteur, des premières coordonnées constitutives du référentiel.

- L'élément vraisemblabilisant de ces fragments de texte vient d'ailleurs renforcer cette hypothèse.

Le paramètre de synthèse permet en outre d'étiqueter le développement algorithmique qui le précède. Ceci est partıculièrement valable dans l'incipit. Dans le déroulement ultérieur du texte, les paramètres de synthèse, coiffant des pans du texte, peuvent constituer des éléments de prévisibilité.

Ces pauses de synthèse dans la lecture correspondent à une mémorisation partielle du texte: la lecture n'a pas pour objet premier de mémoriser, mais enregistrer sous des étıquettes des séries redondantes qui sont réduites graduellement ${ }^{8}$. On l'a vu, l'étiquette peut provenir du texte, et sera de façon privilégiée un paramètre, mais le lecteur peut substituer à celui-ci sa propre formulation.

La mise en place des référents. ou la constitution du référentiel est donc fonction d'une redondance sémantique, elle-mème garante d'une cohérence discursive et narrative.

Tout comme la présence de paramètres dans le texte, le caractère redondant de celui-ci ne doit pas ètre assimilé a la banalité. Si le seuil de redondance est suffisant, la lecture est facilitée ; elle possède alors un caractère automatique, qui demeure fonction des compétences du lecteur. puisque la redondance doit être perçue. 
Un élément non connu comme vigneaux claies dans PAC, par exemple, ne présente pour un lecteur ne possédant pas les coordonnées de la pêche à la morue, aucune redondance et ne peut être sémantisé que par ranger sur et sécher: /support où l'on met le poisson à sécher/.

II y a rupture dans la cohérence du texte, tout comme dans MOE:

IIs s'étaient depuis longtemps rangés du côté du plus fort.

pour un lecteur ne possédant pas les coordonnées lui permettant la substitution: /des parlant français/. Il parviendra à combler ce blanc sémantique par inférence probablement, et le référentiel comportera un élément /français/.

L'absence de redondance peut avoir des conséquences graves lors de la constitution du référentiel. comme le montre l'exemple du bateau dans OCE:

Nous vivons au bord du chemin, le dos au fleuve dans un bateau. Ils l'ont cimenté (...) II s'agit d'un steamer que Ina a trouvé en piochant pour enterrer un de ses chiens.

Si la cohérence-redondance est conforme entre/bateau-chavirer/, et entre/piocher-enterrer-chien/, il n'en va pas de mème entre/trouver un steamer/et /piocher/, surtout après la manipulation qu'exige déjà la proximité /bateau-cimenté/.

II $y$ a donc de fortes chances pour que le lecteur soit obligé, au terme d'une manipulation peut-ètre laborieuse, d'adopter une coordonnée du type /jeu de langage/ qui lui assurera, en référentiel, un décodage plus facile pour la suite du texte.

L'absence de redondance ou de paramètre satisfaisant accroit l'intervention du lecteur sur le texte, et peut bien sûr entrainer des abus d'interprétation. A défaut d'un mode d'emploi propre au texte, le lecteur va s'en fabriquer un.

La nécessité d'un référentiel guidant la lecture appartient à la présomption de sens qui règle toute activité humaine. Le lecteur va suppléer aux apparentes carences du texte, car il existe en regard du programme textuel, programme de lecture, des programmes de lecteur, stratégies de lecture, qui vont modeler le référentiel indépendamment du texte qui n'est plus alors qu'un matériau brut.

Au cours de ce bref examen de trois incipits marquant une certaine diversité, nous avons cherché à montrer le rôle des constantes discursives dans l'élaboration de l'extratexte, et les analogies de fonctionnement qui elles aussi constituent des constantes dans l'activité de lecture.

Le référentiel, espace de travail dans un modèle de performanceréception, est tributaire des paramètres, mais étant donné l'exploitation dont 
ceux-ci font l'objet dans le texte (qu'il s'agisse de Ducharme ou de Thériault) ils ne sont pas a priori garants de la lisibilité, quelque variable qu'elle soit. Ceci nous permet d'écarter la tentation de poser une règle du genre : la lisibilité est directement proportionnelle au nombre de paramètres, car ce serait négliger la cohérence des paramètres entre eux, facteur décisif dans la lecture d'OCE.

En outre, le paramètre, notion dynamique, permet d'écarter le cliché et son corollaire la banalité, qui n'apparaissent dans le modèle de réception que dans l'univers de signification où règne le jugement du lecteur.

Le paramètre permet enfin de lier entre elles et l'exploitation que fait le texte des formes répertoriées du langage (lexicalisées ou en voie de lexicalisation) et la manipulation de ces formes au sein du processus de lecture, notamment dans la coordination propre à la constitution du référentiẹl.

1. Cf. Dubois. J. « Surcodage et protocole de lecture , Poétique 16, Paris : Seuil, 1973, pp. 491-498; Duchet, $C$ «Pour une socio-critıque ou variatıons sur un incipit". Littérature 1. Parıs. Larousse, 1971, pp. 5-14; Hoek, L.H.. Pour une sémiotique du titre, coll. "Documents de travail et pré-publications" $n^{\circ} 20-21$ Série $D$. Urbino: Centro Internazıonale di Semiotica e di Linguistica. Universıtà di Urbıno, 1973: Kristeva. J., Le Texte du roman, Paris-La Haye: Mouton, 1970.

2. Choul, J.-C. Un exemple de programmatique textue/le: Hécate et ses chiens de Paul Morand, mémoire de maîtrise inédit. Universıté du Québec a Montréal, 1977.

3. Le terme de paramètre a été proposé par l'écrivain Jean Paulhan, pour désıgner ces a nouvelles figures»; pour les programmes narratifs, cf. Greimas. A.J. et Courtés, J.. Sémiotique. dictionnaire raisonné de la theorte du langage. Paris: Hachette, 1979.

4. Thériault, Y.. La Mort d'eau. Montréal: Éditions du Jour, 1968, voir appendice.

5. Ducharme, R., L'Océantume. Paris: Gallimard, 1968.

6. Thériault, Y.. La Passe-au-crachin. Montréal: Ėditions du Jour, 1972.

7. L'emploi de sigles permet d'éviter un classement des textes fondé sur un jugement de valeur.

8. On reconnaît dans ces séries redondantes les ısotopıes greımassıennes.

\section{APPENDICE}

\section{Y THERIAULT \\ LA MORT D'EAU}

II filatt les i. II leur mettait un accent circonflexe. II disait les "fiîîilles». II disait aussi «câlice» et cela le marquait. II était Madelinot, un homme des îles depuis toujours qu'ils sont là, ces hommes. 
(Le mot, quand il disait acâlîmices, n'était pas blasphématoire. C'était un soupir, une valeur de soupir, ou de ponctuation. Un point et virgule dans une phrase d'actes quotidiens.)

Lui. Babin, les autres avec lui dans la barque, a cceur de saison de pêche. Primeau Cossett, Lavan, Donaldson. Comeau, parlaient tous ainsi. Ceux qui portaient un nom anglais ne l'étaient pas vraiment, ils s'étaient depuis longtemps rangés du côté du plus fort à Grande Entrée et à Hâvre-Aubert. Ils prononcaient les noms à la française, ils chantaient eux-aussi en parlant.

A cause de la mer, je suppose.

R. DUCHARME:

\section{L'OCÉANTUME}

Elle me demande si j'ai bien appris mes leçons, puis elle me crie d'aller me coucher: comme à un chien. La maitresse d'école a des dents en or plein la bouche. "Mouche-toi donc! Ne laisse pas couler cela comme cela! Tu n'as donc pas de mère pour t'élever? s Nous vivons au bord du chemin, le dos au fleuve, dans un bateau. Ils l'ont cimenté dans le sol, un peu comme une pierre tombale, mais ils ne l'ont pas cimenté tout à fait droit: je souhaite qu'il chavire. II s'agit d'un steamer que Ina a trouvé en piochant pour enterrer un de ses chiens. Ils l'ont peinturé en noir. Ils lui ont fait des fenêtres et des portes de maison. Avant, nous vivions sur une île, juste derrière. Nous sommes partis en voyage et, quand nous sommes revenus, les rats avaient mangé tout le chàteau, sauf la pierre. IIs avaient même mangé les fenêtres et les portes. Parfois, la nuit, toute la terre de l'île se change en rats et les restes du château chancellent. Huit heures, lode; va te coucher! Nonl Elle me flanque une paire de claques a faire tomber le cap Diamant. Je m'entortille comme un bonbon dans mes couvertures. Pendant que peu à peu tout le soleil pris dans la journée sort de mol, je m'imagine que l'école brùle. Je $m$ 'imagine que j'ai des dents en or comme la maîtresse et je suce mes dents.

Y. THERIAULT:

\section{LA PASSE-AU-CRACHIN}

Un jour, un autre jour encore, sans que ce fût là un geste nouveau ou surprenant, Jean qui venait d'amarrer la barque, monta du quai en estacade jusqu'à la maison, se tint dans la porte, et dit a Marie:

- J'ai de la morue, viens.

Alors elle le suivit et se mit au travall à ses côtés. Toute cette journée-lá et le lendemain ensuite, aidés par le matelot Joachim qui était un indien montagnais. Ils dépecèrent le poisson. l'ouvrirent en larges plaques grasses qu'ils placèrent en couches superposées de sel dans les barils poisseux. Plus tard, if faudrait sortir ce poisson et le ranger sur les vigneaux claies où le soleil se chargerait de le sécher. 
C'était une tâche d'homme, une tâche éreintante, sans fin, terriblement monotone, où la blonde Marie tentait une fois encore d'accorder son propre rythme à celui, décidé et preste, de ses deux compagnons. Elle s'en voulait d'avoir cédé une fois de plus. Pendant l'absence de Jean, elle s'était bien promis qu'à son retour. il la trouverait soudain butée et insoumise. S'il se tenait dans la porte et si à son habitude il disait: « J'ai de la morue, viens $\mid \bowtie$, elle resterait assise, elle résisterait. Elle se l'était juré. Et s'il entrait, s'il osait lever la main pour la faire obéir? En toute honnêteté, 